\title{
Be cool: come nasce un'icona culturale
}

a cura di Andrea Bernardelli ed Eduardo Grillo

\section{Testi fatti a pezzi \\ Il culto mediale come ars excerpendi}

\section{Eduardo Grillo}

Accademia di Belle Arti di Napoli, Accademia di Belle Arti di Bologna

eduardogrillo79@gmail.com

$<$ http://www.abana.it/it/docenti/eduardo-grillo $>$

$<$ http://accademiadinapoli.academia.edu/EduardoGrillo>

\begin{abstract}
The article identifies three properties of cultural icons: the recognizability, the ability to generate a community, the tendency of being "reworked". The latter recalls Eco's thought that, in order to become "iconic", a text must be "dismembered" and enjoyed one piece at a time. Later, Eco identified two new requirements: a completely furnished world and the abundance of intertextual archetypes. Our aim is to clarify the nature of these archetypes and the distinctive features of the world represented by the iconic texts. Lastly, we examine the Model Reader of these texts, which seem to invite the readers to play with them, between use and interpretation.
\end{abstract}

\section{Keywords}

Media Cult; Cultural Icon; Intertextual Resonance; Model Reader; Ars Excerpendi

\section{Sommario/Content}

o. Pezzi di testo. Introduzione

1. Culto, icone, sgangherabilità: un approccio pragmatico

2. Modelli e ri-uso. La risonanza intertestuale

3. Il terreno di gioco. Mondi come pre-testi

4. Culto, Lettore modello e Ars excerpendi

Bibliografia 


\section{Ocula}

Vol 21, No 22 (April 2020) • DOI: 10.12977/ocula2020-5

Eduardo Grillo | Testi fatti a pezzi. Il culto mediale come ars excerpendi

\section{o. Pezzi di testo. Introduzione ${ }^{1}$}

Cos'hanno in comune l'ingresso in scena di Darth Vader in Star Wars, accompagnato dal notissimo sottofondo musicale; il dialogo tra Humphrey Bogart e Ingrid Bergman nel finale di Casablanca; l'attacco del discorso di Martin Luther King al Lincoln Memorial; le ultime parole del replicante Roy in Blade Runner; l'incipit di Moby Dick? Salvo il fatto di essere tutte a vario titolo "icone" del Novecento, nulla. Tuttavia, a uno sguardo appena più attento, ci si rende conto che si ha a che fare con parti di testi più ampi. Se non è un caso, improbabile vista l'ampiezza del fenomeno, deve trattarsi di una costante. Così la pensava Umberto Eco che, a più riprese, ha avanzato l'ipotesi che i testi di culto, in particolare i film, si guadagnino il loro status grazie alla propensione a essere "sgangherati". Le parti significative così smontate diverrebbero in seguito oggetto di fruizione e godimento autonomo, generando entusiamo e venerazione. L'ipotesi è così interessante che merita di essere ripresa e sviluppata, ed è quello che si proverà a fare in questo contributo. Tuttavia, la tendenza a essere smembrati non è l'unica caratteristica dei testi iconici, che in effetti attendono ancora una definizione soddisfacente. In primo luogo, dunque, è necessario ridurre, per quanto possibile, l'ambiguità della nozione di "icona". Considerato quanto multiformi siano tali tipi di testo, si preferirà adottare un punto di vista pragmatico, prestando attenzione non solo o non tanto agli aspetti formali, quanto al tipo di relazione che tali testi stabiliscono con i loro fruitori (par. 1). In seguito sarà possibile concentrarsi sui caratteri dei testi "sgangherabili"; seguendo le indicazioni di Eco, si discuteranno quindi la natura degli "archetipi intertestuali" (par. 2) e la foggia del mondo raffigurato dal testo (par. 3). In conclusione (par. 4), si esaminerà il Lettore Modello tipico dei testi di culto, che sembra esibire caratteri peculiari.

\section{Culto, icone, sgangherabilità: un approccio pragmatico}

Ne Il sole nudo, Asimov immagina una sociologia in grado di quantificare con precisione il «differenziale tra scomodità sopportate e privilegi garantiti»; in questo caso, privilegio dell'abbondanza teorica, scomodità dell'indeterminatezza concettuale. Per il culto, basterebbero già le proposte teoriche avanzate nell'ambito di scienze delle religioni per abbandonarsi allo sconforto. Anche restringendo il campo ai fenomeni mediali, le possibilità restano circoscrivibili con difficoltà. ${ }^{2}$ Per le icone culturali non va meglio, sebbene si

1 Devo ringraziare un attento e anonimo revisore per avermi segnalato alcune imperfezioni. La responsabilità di quelle rimaste è, com'è ovvio, soltanto mia.

2 Per esempio, è difficile stabilire in che modo e in che senso una dimensione rituale vada inclusa nella definzione di culto mediale. Il carattere performativo del culto è pertinente in molti casi, ma sembra male adattarsi ai fenomeni ludici tipici della fruizione di alcuni prodotti. D’altra parte sono incerti, in senso generale, i limiti della stessa analogia con l'ambito religioso. Una buona proposta, centrata sulla struttura sintattica del culto, è quella avanzata da Turco nelle conclusioni al suo contributo in questo numero tematico. Qui si seguirà un altro approccio, privilegiando il versante 


\section{Ocula}

Vol 21, No 22 (April 2020) • DOI: 10.12977/ocula2020-5

Eduardo Grillo | Testi fatti a pezzi. Il culto mediale come ars excerpendi

abbia l'impressione che si tratti di materiale più maneggiabile. Si possono certo distinguere icone culturali in senso proprio, rappresentazioni di valori e concetti che riguardano un'intera comunità culturale. Poi icone di culto, che interessano comunità più ristrette, riconducibili ai fenomeni fandom; quindi icone pop, una sorta di via di mezzo quantitativa tra le due precedenti. Ma le vie del culto sono infinite.

Si prendano ad esempio gli "affioramenti" censiti dall'iconologia di Maffesoli (2008). Su "Zidane" si andrebbe forse sul sicuro parlando di icona pop; già su "Harry Potter" vedremmo le prime sovrapposizioni: icona pop, ma certo anche icona di culto. Come regolarsi però con "Che Guevara"? Ecco un caso che forse potrebbe riempire tutte le caselle. Le cose si complicano ancora continuando a scorrere l'indice del libro di Maffesoli; uno accanto all'altro sfilano "Il piccolo principe", "l'Orientalizzazione (della vita quotidiana)", "Cool", "Dioniso (il ritorno)", fino alla "Barba di tre giorni”. Maffesoli assembla la lista, dal vago aroma borgesiano, sostenendo la rinuncia all'atteggiamento iconoclasta degli anni '6o. Si dà quindi il compito di individuare le nuove "idolatrie" senza tentare una delimitazione stringente. Tuttavia, l'impresa rischia di scivolare dall'ecumenismo (comprensibile) all'irenismo (imbarazzante) in mancanza di criteri-guida anche generali.

Con anglosassone senso pratico, Truman (2017) tenta di risolvere la questione pervenendo per via induttiva a una definizione generale di icona culturale. Raccoglie e scompone quindi diverse definizioni avanzate in ambito accademico, proponendo infine questa sinossi: «A cultural icon is a symbol (in the form of person, place or thing) associated with "public good", represented in various forms (mental image, 2D, and $3 \mathrm{D}$ texts), circulating through a variety of networks (social, material, digital) in popular culture, in response to broader modes and contexts of crisis» (Truman 2017: 841). Si tratta di una scelta legittima; ma in tal modo escludono dalla definzione fenomeni più disimpegnati, e abbiamo visto che quando poi si tratta di individuare esempi concreti resta sempre una fondamentale ambiguità. Alcuni usi ludici di icone culturali classiche potrebbero suggerire prudenza prima di stabilire distinzioni nette. Tali fenomeni intervengono spesso aprés coup moltiplicando la forza di diffusione di un'icona, ma potrebbero anche essere coinvolti nella stessa generazione dello status di icona.

Come uscirne? Una via da seguire potrebbe essere quella suggerita più volte da Umberto Eco; visto che la sola semantica non basta, rivolgersi alla pragmatica. Guardando allora all'uso delle icone, si può provare a isolare un nucleo di proprietà, suscettibili di integrazione. In prima approssimazione, si potrebbero quindi rilevare: a) l’immediata riconoscibilità, b) la capacità di generare una comunità, c) la tendenza a essere rilavorate.

a) La riconoscibilità, più o meno estesa, indica in primo luogo una presenza significativa nel contesto culturale; poi il rinvio ai suoi significati possibili in modo quasi automatico. Ossia, le icone sembrano presentarsi come interpretabili in modo magari ampio, ma in apparenza immediata. La riconoscibilità è

pragmatico. 


\section{Ocula}

Vol 21, No 22 (April 2020) • DOI: 10.12977/ocula2020-5

Eduardo Grillo | Testi fatti a pezzi. Il culto mediale come ars excerpendi

insomma un "effetto iconico"; alcune proprietà o relazioni tra proprietà esibite dall'icona culturale appaiono eloquenti e trasparenti, nonostante la potenziale ambiguità. ${ }^{3}$ D'altra parte, la riconoscibilità non implica un'approfondita conoscenza del contesto di origine, né del senso complessivo dell'icona:

Si tratta di figure, luoghi, "oggetti" accomunati dal fatto che hanno assunto un particolare potere simbolico e una presenza durevole nell'immaginario collettivo, divenendo parte di un patrimonio simbolico comune e trasversale da cui attingono il linguaggio giornalistico e quello pubblicitario, la comunicazione colta e il parlare comune. [...] Si tratta di figure mendacemente familiari, note anche se il più delle volte non propriamente conosciute. Oggetto di una conoscenza diffusa e generica, di un uso generalizzato e spesso improprio [...] [la] leggibilità immediata garantisce un effetto immediato di riconoscimento [...] Il tessuto di fondo di queste icone si trasforma [...] anche profondamente, ma conserva sempre un profilo di fondo e delle caratteristiche che le rendono immediamente riconoscibili. (Fiorentino 2009, pp. 9-11)

L'estrema flessibilità semantica è associata da Fiorentino a un'azione costante nei vari contesti storico-culturali. I due aspetti non sono in contraddizione; l'icona resta sempre riconoscibile ed efficace proprio perché elastica e adattabile, ambigua e trasparente al contempo.

b) Con la capacità di generare una comunità si recupera l'aspetto cultuale. Con "culto" indicheremo quindi la disponibilità a riconoscere, dedicarsi, discutere e godere di un testo, un'immagine, una persona, un luogo sentendosi al contempo parte di un gruppo che nutre la stessa "devozione". La "comunità" generata da un'icona culturale sarà di taglia diversa e varia struttura: potrà essere un intero Paese (simbolo identitario nazionale), una "setta" (happy few), un agglomerato i cui membri saranno per lo più ignari della reciproca identità (gli "adepti" di una serie tv). In tal senso, sembra possibile ampliare la portata delle riflessioni di Altman (1999) sui generi cinematografici a proposito di "comunicazione laterale" e "comunità costellate". Infatti, l'interazione più o meno diretta tra fan o seguaci di un testo o un personaggio iconico è in grado di dar vita a comunità coagulate soltanto dal coinvolgimento generato da quel testo. I membri di tali comunità sarebbero uniti insomma soltanto da "atti di immaginazione" e non dalla necessaria condivisione di un ampio spettro di interessi e valori.

c) La tendenza delle icone culturali a essere trasformate e riutilizzate è stata sottolineata di recente da Ortoleva, nell'ambito della sua riflessione sui miti a "bassa intensità". Tali narrazioni, più accessibili rispetto ai miti classici, "vengono continuamente ri-narrate da un medium all'altro [fino a entrare] nel circuito della conversazione» (Ortoleva 2019: 32). In modo più incisivo,

3 Tuttavia in ragione di «un'associazione di idee generali, in base a una legge culturale consolidata, in base a regole culturali che determinano e arricchiscono e in qualche modo inchiodano l'oggetto alla sua forma culturale e iconica di una convenzione o "legge"» (Ferraresi, "Dall'icona all'icona culturale», in questo numero tematico, par. 4). Si è insomma in piena Terzità. Si tratterebbe dunque di ipoicone simboliche; per un approfondimento, si rimanda al citato articolo di Ferraresi. 


\section{Ocula}

Vol 21, No 22 (April 2020) • DOI: 10.12977/ocula2020-5

Eduardo Grillo | Testi fatti a pezzi. Il culto mediale come ars excerpendi

Fiorentino descrive le icone come «elementi significativi di un "canone selvaggio", cioè fatto di frammenti di "narrazioni" (racconti mitici, letterari cinematografici, ma anche pittorici e musicali; rappresentazioni collettive o pratiche culturali, rituali, discorsive, ecc.) che, emancipati dal loro contesto di origine, agiscono indipendentemente da esso in una pluralità di altri contesti» (Fiorentino 2009: 9). ${ }^{4}$

Il riferimento alla frammentarietà, all'indipendenza e all'autonomia delle icone culturali rammenta le riflessioni di Umberto Eco sui film di culto, avanzate in una serie di interventi più o meno occasionali (Eco 1977, 1985, 1994, 1999). Tali riflessioni da un lato fanno sistema con le considerazioni di Ortoleva e Fiorentino, dall'altro pongono la propensione a sganciarsi dai contesti originari alla base stessa della nascita delle icone culturali. Non soltanto un tratto definitorio dunque, ma un meccanismo o un processo di generazione.

Difatti, secondo Eco, un film come Casablanca, insoddisfacente rispetto ai parametri di una critica esigente, sarebbe oggetto di un entusiasmo transgenerazionale non nonostante, ma proprio essendo un patchwork di situazioni stereotipe, soprattutto di natura archetipica: «Casablanca non è un film, è tutti i film» (Eco 1977: 142). Il punto centrale è che la mancanza di organicità e la sovrabbondanza di cliché indurrebbero gli spettatori a "sgangherare" il film per gustare le singole scene, disseminandone luoghi e tempi di fruizione e sollecitando una disordinata catena di citazioni. Il film beneficerebbe dunque à rebours della sua onnipresenza "distributiva" acquistando una sorta di aura intertestuale; in tal modo, Casablanca diverrebbe un'icona e insieme una riserva di icone di taglia minore. ${ }^{5}$

Si noti che l'equazione tra modesto valore estetico e culto tributato sarebbe necessaria solo per i film, poiché un film ben realizzato lascerebbe secondo Eco un ricordo unitario, minimizzando il ruolo dei brani notevoli. Inoltre, la difficoltà di recuperare le singole scene (Eco scriveva quando DVD e piattaforme online con funzione segnalibro erano di là da venire $)^{6}$ non sembra conge-

4 La nozione di “canone selvaggio" (wilder Kanon) è tratta da Eibl (1998; 60-77). Fiorentino (2009) ha il merito di aver esteso, secondo modalità che paiono legittime e produttive, la portata di un concetto nato in riferimento alla letteratura.

5 Se l'approccio di Eco ben si adatta alle opere di finzione (è nato per questo), con gli opportuni aggiustamenti potrebbe applicarsi in modo efficace, sebbene non esclusivo, anche a oggetti e personaggi del mondo reale. Ad esempio, nel caso di persone in carne e ossa si tratterebbe di individuare gesti, frasi e comportamenti suscettibili di essere "sgangherati" dal contesto d'origine e citati e trasformati più volte senza riguardi filologici. In effetti, è quel accade spesso nel processo di generazione di molti meme.

6 Tuttavia, Guagnelini e Re (2007) argomentano a favore dell'attualità della distinzione tra fruizione cinematografica e letteraria; la "legge dello scorrimento" tipica della visione su pellicola viene sì meno con la diffusione di nuovi supporti, ma «il vincolo tecnologico rimane. [...] occorrono, quanto meno, un lettore DVD e uno schermo. [...] nei supporti digitali è stata introdotta la funzione del "segnalibro": ma se chiunque è abituato a fare un'orecchia all'angolo di un pagina, lo stesso non si può dire dell'abitudine (che richiede una certa dimestichezza con i software di lettura di file video) di marcare specifiche sequenze da recuperare più rapidamente in un secondo momento» (167). Dal libro di Guagnelini e Re sono passati più di dieci anni; in linea 


\section{Ocula}

Vol 21, No 22 (April 2020) • DOI: 10.12977/ocula2020-5

Eduardo Grillo | Testi fatti a pezzi. Il culto mediale come ars excerpendi

niale a una fruizione "citazionale". Invece, i testi letterari consentirebbero ai lettori di sganciare singole parti in ogni caso, poiché i brani di un testo scritto si possono sempre recuperare con facilità, nel caso di un capolavoro come nel caso di un fallimento artistico. A ogni modo, forse proprio la differenza rilevata tra culto cinematografico e letterario ha stimolato Eco a stabilire una distinzione più generale tra sgangheratezza e sgangherabilità (Eco 1999). In sostanza, sgangherate sarebbero, com'è ovvio, opere sconnesse e disorganiche, di cui Casablanca è un campione. Sgangherato significa anche sgangherabile; ma opere ben riuscite, dunque non sgangherate, possono essere sgangherabili purché dotate di specifici caratteri. In (Eco 1977) si fa riferimento a un elevato grado di complessità testuale (ad esempio la Divina Commedia); in (Eco 1985), più sistematico, si discutono in dettaglio tre "requisiti" testuali.

Il primo riguarda il mondo raffigurato dal testo. Secondo Eco, dovrebbe essere completo e dettagliato, così che «fans can quote characters and episodes as if they were part of the beliefs of a sect, [...] a world about which one can play puzzle games and trivia contests, and whose adepts recognize each other through a common competence» (Eco 1985: 3). In sostanza, un mondo "ammobiliato" in grado di garantire una sorta di "abitabilità" e un alto grado di interazione da parte dei fruitori. Il secondo riguarda invece la natura degli elementi testuali che tendono a essere memorizzati e poi isolati dal testo. Il fatto che siano cliché o topoi non è sufficiente; devono avere un certo "archetypal appeal". Eco non pensa ad archetipi junghiani,7 quanto piuttosto a fenomeni "laici" di risonanza intertestuale. Il terzo e ultimo carattere è proprio una struttura testuale priva di «una coerente filosofia della composizione», in modo da sollecitare il lettore a smontarne le parti. Tuttavia, nel tentativo di porre le basi di un approccio valido allo stesso modo per "testi iconici" sia sgangherati sia sgangherabili, si dovrebbe parlare piuttosto di segnali di modularità, questione che, per ragioni di spazio, non verrà discussa qui.

\section{Modelli e ri-uso. La risonanza intertestuale}

A proposito degli elementi testuali "sgangherabili" (cliché, topoi, ecc.), Eco distingue con acutezza tra «more or less universal narrative functions à la Propp, visual stereotypes like the Cynic Adventurer, and more complex archetypal situations like the Unhappy Love [...] the duel between the sheriff and the bad guy or the narrative situation in which the Hero fights against the Villain and wins; as well as more macroscopic textual situations, such as the story of the vierge souillée or the classical scene of the recognition", tracciando quindi un confine tra due insiemi più generali: «We could distinguish

generale la distinzione potrebbe essere ancora valida, ma la questione non è centrale per il nostro discorso.

7 In Grillo (2012) si trattavano gli elementi "sgangherabili” presentando anche gli archetipi junghiani; ma in quel contesto, per lo più didattico, l'obiettivo era familiarizzare il lettore con una nozione generale di archetipo. Nel paragrafo 2 si tenterà invece di discutere quale senso può avere, in prospettiva semiotica, l'espressione "appeal archetipico" utilizzata da Eco. 


\section{Ocula}

Vol 21, No 22 (April 2020) • DOI: 10.12977/ocula2020-5

Eduardo Grillo | Testi fatti a pezzi. Il culto mediale come ars excerpendi

between stereotyped intertextual frames (for instance, the Drunkard Reedemed by Love) and stereotyped intertextual iconographical units (for instance, the Evil Nazi)» (Eco 1985: 4-5). La suddivisione proposta da Eco discrimina in effetti tra entità testuali di taglia e natura diversa, laddove molti esempi dei formalisti russi appaiono più ondivaghi. Il ricorso alla nozione di frame (sceneggiature comuni e intertestuali, Eco 1979, pp. 79-85) serve a rendere conto di tutte queste unità testuali, nonché a non compromettersi con le infinite connotazioni del concetto di archetipo.

Infatti, come si è accennato al paragrafo precedente non basta che tali unità siano già note; devono esibire un "appeal archetipico":

"A suspect who escapes a pass control and shot by the police" is undoubtely an intertextual frame, but it does not have a "magic flavor". [...] Let us define as "magic" those frames which, when appearing in a movie, and when separated from the whole, transform this movie into a cult movie. [...] Let us call these latter intertextual archetypes. The term "archetype" here does not pretend to have any particular psychoanalitic or mythic connotation, but serves only to indicate a pre-established and frequently re-appearing narrative situation that is cited or in some way recycled by innumerable other texts, and provokes in the addressee a sort of intense emotion accompanied by the vague feeling of a déja vu that everybody yearns to see again. I would not say that an intertextual archetype is necessarily "universal". It can belong to a rather recent textual tradition, as it happens with certain "topoi" of slapstick comedy. It is sufficient to consider it as topos or standard situation that comes to be particularly appealing to a given cultural area or historical period. (Eco 1985: 5)

È chiaro l'intento di ricondurre nell'alveo socio-culturale e testuale nozioni dal potenziale semantico intrattabile, ma resta una sostanziale vaghezza nella definizione di un "aroma magico" degli archetipi intertestuali, riecheggiata diversi anni dopo nella descrizione del mito come "quello che resta" (Spaziante 2006). Si pone insomma il problema di "laicizzare" la nozione di archetipo individuando però almeno alcune caratteristiche testuali, al di là del pur necessario riferimento all'emozione veicolata e al fascino esercitato su una comunità di interpreti.

Eco ne individua almeno una, ossia la capacità degli archetipi di dar vita a intere storie (cfr. Eco 1977: 139). In effetti, un semplice topos non sembra avere la forza, da solo, di generare interi intrecci. Tale caratteristica è stata evidenziata anche da Meletinskij (1994), che condivide l'approccio storico-culturale di Eco, in deciso contrasto con i «riduzionismi psicologici e mitico-rituali». Dall'approfondito esame di Meletinskij si può trarre un altro carattere degli archetipi, cioè la tendenza a subire trasformazioni. Sembra infatti che semplici frame o situazioni stereotipe mostrino una maggiore rigidità, mentre gli archetipi avrebbero la natura, come dichiara l'etimologia, più di modelli, di "stampi" e meno di unità combinatorie. Del resto, la dimensione intertestuale degli archetipi non è dovuta soltanto alla frequenza con cui ricorrono, ma alla propria qualità connettiva. Frye (1957), pur risentendo dell'approccio mitologico e psicanalitico, ha espresso questo aspetto con efficacia, definendo 


\section{Ocula ${ }^{22}$}

Vol 21, No 22 (April 2020) • DOI: 10.12977/ocula2020-5

Eduardo Grillo | Testi fatti a pezzi. Il culto mediale come ars excerpendi

l'archetipo un «simbolo che coniuga una poesia ad altre poesie e serve a unificare e integrare la nostra esperienza letteraria» (Frye 1957: 128, tr. it.). Al di là delle differenze terminologiche, la definizione di Frye è preziosa, poiché mette l'accento sulla natura di dispositivi intertestuali degli archetipi. Tale proprietà sarebbe dovuta, riassumendo, al carattere di modello degli archetipi. Proprio questa "elasticità", d'altra parte, giustifica le numerose riflessioni sulla significatività antropologica degli archetipi, al di là delle energie psichiche coinvolte che sembrano cadere al di fuori dei limiti di un approccio testuale.

Per cominciare a chiarire meglio la qualità "magica" degli "archetipi intertestuali”, queste prime evidenze devono essere integrate guardando al modo in cui essi si offrono agli interpreti. Un buon punto di partenza sembra tracciare una parentela, mutatis mutandis, tra le modalità di presentazione degli archetipi intertestuali e il discorso di ri-uso individuato da Lausberg (1963: 16-17), sviluppato in ambito letterario da Brioschi e Di Girolamo (1984: 9-16). In effetti, la nozione viene messa in gioco dai due autori nel tentativo di individuare i caratteri che definiscono un'opera letteraria, in particolare le sue condizioni pragmatiche. Ma le loro riflessioni, tenuto conto della diversa finalità, disegnano un griglia che ben si presta alla descrizione del fenomeno indicato da Eco.

Il discorso di ri-uso riguardava in Lausberg (1963) leggi giuridico-sacrali o profano-sacrali, formule performative e di ratifica, discorsi fissati di importanza collettiva. La rielaborazione di Brioschi e Di Girolamo assegna ai tre campi un senso più generale, distinguendo scritture sacre, leggi e testi letterari, quest'ultimi accanto ai testi filosofici e scientifici. Un testo soggetto a ri-uso avrebbe poi tre aspetti principali: un dominio di applicazione, un'identità, un'asimmetria tra i soggetti della comunicazione (cfr. Brioschi e Di Girolamo 1984: 11). Il dominio è l'ambito di applicazione del ri-uso di un testo, che a differenza del testo di consumo deve essere valido e pertinente in un ampia serie di casi. L'identità è un tratto definitorio; se fosse compromessa, verrebbe meno lo stesso ri-uso. L'asimmetria è propria ai testi di ri-uso, poiché una legge, un testo sacro o un testo letterario stabiliscono un rapporto ineguale tra l'emittente e il ricevente: una legge prevede l'obbedienza, un testo sacro la fede $\mathrm{o}$ al più l'esegesi, un testo letterario la lettura o la citazione; ma in nessun caso questo tipo di testi autorizzano una "risposta" su uno stesso piano assiologico. In relazione al campo letterario poi, i tre aspetti conoscono un'ulteriore precisazione; poiché sono i soli che interessano qui, si prenderanno in esame i primi due.

a) Per quanto riguarda il dominio, se «non è difficile immaginare a che cosa si applica una legge o una proposizione scientifica», è molto meno agevole stabilire a quali situazioni o esperienze possa applicarsi un testo letterario. Tuttavia, «non sarà vero [...] che una poesia o un racconto sono destinati appunto a "creare" nella mia mente l'esperienza in oggetto? L'ipotesi sembra più che plausibile [...] io sono chiamato a riprodurre nella mia mente ciò che il testo denota ed esemplifica, e che proprio ciò costituisce l'esperienza "dominata" dal testo stesso» (Brioschi e Di Girolamo 1984: 13).

b) Per quanto riguarda l'identità, alla sua conservazione nel ri-uso «è con- 


\section{Ocula}

Vol 21, No 22 (April 2020) • DOI: 10.12977/ocula2020-5

Eduardo Grillo | Testi fatti a pezzi. Il culto mediale come ars excerpendi

nessa, appunto, la questione della sua insostituibilità. Il ri-uso letterario valorizza più di ogni altra forma di ri-uso gli aspetti esemplificazionali del testo; negli altri casi [leggi, testi sacri, teorie scientifiche] prevalgono invece altri motivi d’insostituibilità» (Brioschi e Di Girolamo 1984: 14).

Ora, si è visto che gli archetipi individuati da Eco si distinguono dai semplici topoi per la loro natura di dispositivi intertestuali e per la maggiore capacità di generare intrecci. Sembra ragionevole allora individuare in un esteso dominio la ragione di tale produttività. Gli archetipi avrebbero insomma maggiori potenzialità nel generare esperienze ricche, più duttili e quindi "applicabili" a una più larga sfera di interessi, idee e sviluppi narrativi. Il fatto che siano in grado di "dominare" un ampio ventaglio di situazioni dipende d'altra parte da un carattere che caratterizza anche la sua identità nel ri-uso, ossia la forza esemplificativa. Si tratta di intendere con esemplificazione la capacità di "incarnarsi" in una quantità indeterminata di realizzazioni testuali restando sempre riconoscibile. Insomma, una maggiore capacità di essere esemplificato ${ }^{8}$ rispetto ai semplici motivi, ai topoi o agli stereotipi, mantenendo immutata identità e inesauribilità.

È chiaro a questo punto perché un testo ricco di archetipi, sia pure senza gli eccessi di Casablanca, possa risultare "sgangherabile". Il punto è, come si è visto, che l'archetipo non è soltanto un'entità tematica, ma un modello. Un motivo suggerisce una struttura sintattica precisa degli eventi; un archetipo evoca un mondo, convoca un'intera tradizione testuale, limitandosi a fornire istruzioni e suggestioni più generali, ma per questo più produttive.

\section{Il terreno di gioco. Mondi come pre-testi}

Eco (1985) non si diffonde a proposito del mondo disegnato dal testo; in effetti, si limita a indicare la necessaria completezza del suo "arredamento". 9 Tuttavia, è interessante la relazione stabilita tra questa caratteristica e l'elevato grado di interazione da parte dei fruitori.

Per cominciare, sebbene la terminologia usata da Eco rinvii d'emblée allo strumentario teorico dei mondi possibili, ricorrervi non sembra pertinente. Infatti, è nota la posizione dello stesso Eco: in relazione all'universo della narrazione, i mondi possibili vanno intesi come costrutti culturali, ammobiliati, descrivibili attraverso matrici di proprietà che non consentono il calcolo (Eco 1979, cap. 8). ${ }^{10}$ Quel che è più importante, Eco asserisce con decisione che « $\mathrm{E}$ utile usare la nozione di mondo possibile quando ci si riferisce a uno stato di

8 Solo a questo livello, molto generale, si può intravvedere un parallelo con l'esemplificazione di Goodman, laddove essa non comporta per l'opera il denotare, ma l'essere denotata dai predicati che gli si possono applicano (Goodman 1968; 1978).

9 Approvata da Eco, la traduzione di Simona Mabrini in Guagnelini, Re (2007) recita «universo completamente autosufficiente» (173). Si possono considerare le due espressioni intercambiabili, sebbene alla luce di quel che si dirà la versione italiana sembrerebbe più vantaggiosa.

10 Tali assunzioni marcano la differenza con la nozione omonima utilizzata in logica modale. 


\section{Ocula}

Vol 21, No 22 (April 2020) • DOI: 10.12977/ocula2020-5

Eduardo Grillo | Testi fatti a pezzi. Il culto mediale come ars excerpendi

cose, ma soltanto se occorre confrontare almeno due stati di cose alternativi» (Eco 1990: 198). E non è questo il caso, poiché si tratta di considerare il "mondo possibile" descritto dal testo in se stesso, senza che sia necessario sollevare la questione dell'accessibilità tra mondi.

Piuttosto, la breve descrizione di Eco sembra accostare i due modelli di "testo come mondo" e di "testo come gioco" che vengono invece spesso contrapposti. In effetti, il primo modello sembra implicare un effetto immersivo sul fruitore, mentre il secondo richiama piuttosto l'interazione: un mondo si abita, un gioco invita alla partecipazione. Un'altra differenza riguarda l'atteggiamento del fruitore nei confronti delle strategie testuali. Prestando attenzione ai mezzi espressivi del testo non sembra plausibile sprofondare immemori in un mondo fittizio, mentre per giocarvi bisogna essere consapevoli delle regole (anche per trasgredirvi) e degli strumenti a disposizione per compiere le proprie mosse. Nel caso della coppia mondo-immersione, la "manifestazione lineare" del testo (Eco 1979) sarebbe dunque percepita come trasparente; spessa e opaca nel caso della coppia gioco-interazione (cfr. Ryan 2015: 117-118 et passim). ${ }^{11}$

La distinzione appare forse un po' troppo netta; la stessa Ryan (2015, capp.9-10) si impegna a riconciliare i due modelli. In ogni caso, si dirà che se non per tutti i tipi di testo, almeno nel caso dei testi di culto sembrano necessarie sia una forma di immersione (quindi un mondo completo che inviti a ritornarvi), sia una forma di interazione (una stuttura che faccia da supporto per le iniziative del fruitore).

Ora, sembra piuttosto ovvio che ciò che rende tale un mondo non è certo una collezione di frammenti a malapena tenuti insieme, quanto invece «a connected set of objects and individuals, a habitable environment, a reasonably intelligible totality for external observers, and a field of activity for its members» (Ryan 2015: 63). Per tornare all'esempio-paradigma di Eco, Casablanca ha in effetti una cartografia, forse appena abbozzata, ma chiara a sufficienza. Mostra un ambiente molto popolato, in relazione con altri distretti del mondo (sullo sfondo ci sono Lisbona porto franco e la Francia di Vichy); c'è un passato plausibile, anche se solo evocato. Insomma, la mancanza di coesione è narrativa, ma il mondo in cui hanno luogo gli eventi è privo di contraddizioni. Esso è coerente quanto basta per dare un rilievo non problematico a luoghi notevoli; tornandoci con il ricordo, lo spettatore ha cioè la possibilità di fare delle soste, di seguire il cammino dei personaggi, di attenderli prima che il loro destino si compia. Sebbene non sia cesellato, il mondo di Casablanca è sempre disponibile all'esplorazione, accogliente e visitabile a volontà.

Da tali considerazioni emerge un modello di immersività non solo o non tanto "verticale", profondo e limitato alla singola sessione fruitiva, quanto "orizzontale", magari anche più superficiale, ma ripetuta nel tempo. Si tratta della capacità del testo di essere evocato innumerevoli volte nella memoria e

11 Non va dimenticato che Ryan si interessa soprattutto di videogame, nonché di programmi di intrattenimento che sfruttano la realtà virtuale. Tuttavia, la sua discussione sui modelli di testo ha più ampia portata. 


\section{Ocula}

Vol 21, No 22 (April 2020) • DOI: 10.12977/ocula2020-5

Eduardo Grillo | Testi fatti a pezzi. Il culto mediale come ars excerpendi

nella discussione con gli altri membri della "setta". In sostanza, coerenza e ricchezza del "mondo" accendono la fantasia e nutrono la memoria, invitando a nuove esplorazioni del testo. A queste caratteristiche vanno aggiunti due tratti che sembrano responsabili della continua disponibilità all'approfondimento narrativo, ossia il continuo va-e-vieni da e verso un mondo fittizio.

a) Si è già accennato a densità di oggetti e personaggi, nonché a spostamenti nello spazio e a luoghi privilegiati. Va inoltre precisato che, affinché uno spazio rappresentato consenta davvero la continua esplorabilità, è necessario ottenere un "effetto di profondità" (Cavicchioli 2002), nonché una buona resa di luoghi riconoscibili e ben caratterizzati. Il primo è essenziale per la costruzione efficace e credibile di uno spazio descritto o comunque rappresentato:

Da un lato, si tratterà di mettere in scena dei processi avviati da un soggetto che tramite le sue azioni e i suoi movimenti evidenzia lo spazio come luogo della profondità; dall'altro, la stessa convivenza, all'interno di un medesimo campo visivo, di più oggetti, ci restituirà quello spazio come luogo articolato di connessioni, che liberano appunto un effetto di abitabilità e percorribilità e, ancora un volta, di profondità. (Cavicchioli 2002: 236).

Movimento, densità e l'effetto di profondità che ne consegue sono insomma necessari a generare, se non un quadro preciso dello spazio d'azione dei personaggi, almeno una buona descrizione di alcuni luoghi notevoli. Non è infatti indispensabile una mappa dettagliata dell'intero mondo; l'importante è che alcuni luoghi siano credibili e ben tratteggiati. Pensare a quei luoghi in cui avvengono prove decisive o trasferimenti significativi potrebbe essere fuorviante. Sembra questa una delle differenze tra un testo anche di successo e uno di culto; il primo può anche dedicare i maggiori sforzi descrittivi (e in genere lo fa) allo spazio che ospita il redde rationem o gli spostamenti irreversibili e simbolici. Il secondo presta uguale attenzione, in genere, a spazi eccezionali e comuni, con l'effetto ulteriore di non costruire soltanto uno spazio (space), ma un luogo (place). Una casa alla quale tornare, facilitando così l'immersività nel tempo che si è chiamata "orizzontale". Tale carattere appare ovvio per i testi seriali, ma è ipotizzabile e da verificare una sua più generale validità.

b) Il continuo approfondimento narrativo sembra stimolato anche dalla gestione del tempo, parte integrante del mondo rappresentato. Un testo ricco di "disgiunzioni di probabilità"12 e "segnali di suspense" (Eco 1979, cap. 7) invita a supplementi d'ispezione a maggior titolo rispetto a testi in cui la tensione narrativa giochi un ruolo minore. È infatti più facile rievocare le vicende costellate di momenti di tensione, ma non è necessario che l'incertezza sia davvero genuina. Approcci più ironici e insistiti sono anzi campioni paradigmatici di una frequentazione anche ossessiva.

12 Le quali, sollecitando delle "passeggiate inferenziali", renderebbero pertinente il ricorso ai mondi possibili, ma riferendosi all'accessibilità tra stati alternativi della fabula immaginati dal lettore, e non in relazione al mondo evocato dal testo tout court. 


\section{Ocula}

Vol 21, No 22 (April 2020) • DOI: 10.12977/ocula2020-5

Eduardo Grillo | Testi fatti a pezzi. Il culto mediale come ars excerpendi

Con tutte queste considerazioni a proposito del mondo rappresentato non si è ancora nel regno esclusivo dei testi di culto. Tuttavia, parlando di approfondimento narrativo o frequentazione ossessiva si è già intravvisto il secondo aspetto indicato da Eco, la possibilità di interazione e di gioco con il testo, determinante per l'affermazione del culto. Si è detto che i due modelli gioco $e$ interazione/mondo e immersione sono spesso presentati in opposizione. Ma in effetti, un mondo ricco di luoghi ben descritti e riconoscibili; punteggiato di momenti di tensione narrativa; propiziando l'emergenza della dimensione orizzontale dell'immersione offre un supporto ideale alle manipolazioni e alla citazione. Il punto è che se di gioco si tratta, non sarà tanto per via di regole esplicite e di mosse consentite. Si pensi ai giochi di ruolo: un mondo in cui sono descritti i luoghi fondamentali (ognuno con un contegno emotivo suo proprio), che consente di tornare indietro e prendere un'altra strada, fa da sfondo a una serie di azioni immaginabili secondo alcune regole più o meno dettagliate. Il mondo di un testo di culto fa insomma da "plancia" o "tabellone" per le scorrerie dei fruitori. Non si tratta più di un "testo", ma di un "pre-testo" dal quale, alla lettera, prendere le mosse. La differenza con i giochi di ruolo, o con qualunque altro gioco, è che l'atteggiamento dei fruitori non è spesso quello del giocatore puro. Il fruitore che torna al mondo di un testo di culto sembra più un appassionato di partite leggendarie; le ripete, ne recita i passaggi salienti, ne discute con altri appassionati, fino ad ampliarne i significati o idearne versioni alternative. È un storico eccentrico che, quando si cita, esige pieno rispetto filologico; ma si permette piena libertà di manipolazione per innestarle in contesti nuovi e più attuali.

\section{Culto, Lettore Modello e ars excerpendi}

Il fatto che lo stile fruitivo sollecitato dai testi di culto attribuisca loro un carattere di "pretesti" induce una serie di considerazioni ulteriori. Innanzitutto, sembra suggestivo considerare l'attività del fruitore una forma rinnovata di un'arte antica e veneranda, sebbene con scopi differenziati.

Difatti, l'uso di estrarre brani dai testi (per citare, assemblare florilegi o accumulare materiale di riflessione) è sempre esistito. ${ }^{13}$ Quest'uso ha il nome savant di ars excerpendi, una tecnica quindi, impiegata da studiosi e lettori di tutte le epoche. Studiosi e lettori; infatti, sebbene il termine rinvii a una pratica utile all'approfondimento e l'assimilazione dei testi, copre di fatto anche l'atteggiamento appassionato di una vasta platea di fruitori che "tirano a sé" il testo trascegliendo e gustando brani rilevanti. Si intravvede così, al

13 Cevolini (2006) ne censisce esempi fin dall'antichità, per poi mettere in rapporto il tema dell'oblio minaccioso con l'affermazione della tecnologia della stampa. Si tratta della presa di coscienza, davanti all'inedita sovrabbondanza delle fonti, che si è entrati nell'era della dimenticanza (Cfr. anche Eco 2007: 86-96). Il legame tra ars excerpendi e oblio sarebbe da approfondire anche in relazione al culto, per esempio indagando in quale misura l'affermarsi di un'icona culturale non possa servire per seppellire sotto un velo ideologico, o al contrario recuperare alla memoria, alcuni temi o eventi importanti in seno a una società. 


\section{Ocula}

Vol 21, No 22 (April 2020) • DOI: 10.12977/ocula2020-5

Eduardo Grillo | Testi fatti a pezzi. Il culto mediale come ars excerpendi

di là della semplice attività di sottolineatura e annotazione che accompagna la lettura, un'ars legendi come ars excerpendi (Minzetanu 2012) che assume quasi i caratteri di una "poetica della ricezione". Tale "poetica" si regge su un atteggiamento indiretto nei confronti del testo:

La lecture citationnelle $[\ldots]$ n'envisage pas le texte $[. .$.$] comme une système, comme$ une hiérarchie de structures, comme un organisme où tout est important, où tout se répond et où chaque élément contribue à la cohérence de l'ensemble, mais comme un jardin où l'on vien cueillir les meilleurs fleurs, les meilleurs arguments et les plus belles phrases; [...] Ce type de lecture (dans lequel on peut avoir l'impression que le texte est là comme prétexte ou comme contexte, pour rendre possibile l'avènement d'une phrase), fait de la Phrase l'object même de la littérature [...] Il ne s'agit pas tellement de comprendre la phrase [...] mais de la vivre comme un «événement figural», comme un «jeu de tensions » et comme une «expérience temporelle» [...] L'enjeu fondamental de ces phrases est leur disponibilité en vue d'une possible réutilisation (Minzetanu 2012: 32).

Si tratta di un'attitudine diffusa, in stretta correlazione con i fenomeni di culto, ma più ampia, che ha già stimolato parecchie riflessioni. Tra le più acute, quelle semiotico-estetologiche di Calabrese che, grosso modo in contemporanea alle riflessioni di Eco, ha inserito dettaglio e frammento tra i principi della sensibilità neobarocca. ${ }^{14} \mathrm{Si}$ tratta di due modalità di conoscenza, produzione e apprezzamento estetico di prodotti culturali che presuppongono stili cognitivi differenti; il primo è infatti a carico di un soggetto che esalta una parte dell'intero, il secondo a carico del tempo che lascia al soggetto il compito di ricostruire un intero ormai perduto (cfr. Calabrese 1987: 108-125, ed. cons.). L'ars excerpendi della lettura citazionale ricorda l'operazione dettagliante indicata da Calabrese; tuttavia lascia pensare a un misto, laddove i "dettagli" ricavati dal soggetto sono poi diffusi come frammenti. In effetti, si è visto che al limite il fruitore potrebbe anche non conoscere il testo di partenza, e molto spesso così accade, come ha evidenziato Fiorentino (2009). Ora, considerare un testo come pretesto, approcciarlo come un insieme non sistematico, valorizzarne i dettagli e diffonderli come frammenti sono disposizioni che fanno dell'attività di interpretazione «an impertinent ride on the literary preserve that takes away only those things that are useful or pleasurable to the reader [...] De Certeau's "poaching" analogy characterized the relationship between readers and writers as an ongoing struggle for possession of the text and for control over its meaning» (Jenkins 1992: 24-25). Il che non è senza conseguenze per una teoria semiotica dell'interpretazione.

Infatti, l'incursione è impertinente certo nei confronti degli autori, ma soprattutto nei confronti dei "diritti del testo". Il lettore "nomade" dedito al "bracconaggio" di De Certeau (1980), il fan partecipativo e rivaleggiante di Jenkins si mostrano indisponibili a "farsi usare" dal testo (Eco 1979: 58). Se

14 Per un primo esame delle relazioni tra estetica neobarocca e sgangherabilità, in relazione a testi seriali, si vedano le note in Grillo (2016). 


\section{Ocula}

Vol 21, No 22 (April 2020) • DOI: 10.12977/ocula2020-5

Eduardo Grillo | Testi fatti a pezzi. Il culto mediale come ars excerpendi

il Lettore Modello consiste nelle istruzioni implicite che un testo impartisce per la propria interpretazione (Eco 1979), tali "lettori" procedono invece senza scrupoli filologici o "ecologici", prelevando souvenir e al limite sfidando il testo. Il punto è che in tali casi si assottiglia il confine tra uso e interpretazione (Eco 1979: 59-60 ss.; Eco 1990: 32-34 ss.), e in modo insidioso, poiché si tratta di pratiche intersoggettive. Ora, una teoria dell'interpretazione non può cedere le armi all'eventuale sconsideratezza degli interpreti; tuttavia, non si può ignorare che i comportamenti concreti si attestino spesso ai suoi margini. Ma difatti, Eco non ha inteso stabilire un'opposizione dai confini netti. Piuttosto, si tratta di immaginare un progressivo digradare che vada dalla fedele adesione all'intentio operis a esercizi di appropriazione e ri-uso, fino a drammatiche incomprensioni:

più che segnare un'opposizione, la coppia uso/interpretazione indica due momenti di un continuum, in cui una certa fascia mediana è più "normale", perché più legittimabile, sia da elementi testuali che dalla comunità degli interpreti, mentre le altre sono più estreme e forse per questo anche più discutibili e interessanti. L'uso è in qualche modo sempre collaterale - sotto (misunderstanding), sopra o accanto (metalettura) le righe del testo. (Pezzini e Sabucco 2007: 155) ${ }^{15}$

Lungo questo continuum, la pratica di estrazione, smontaggio e bricolage che secondo Eco individua il culto sembra collocarsi a mezza via tra l'interpretazione in senso proprio e un uso davvero spregiudicato: può dar vita a sfide mnemoniche, prassi di commento più o meno ironico, innesti tra universi narrativi e vere e proprie continuazioni o riscritture. In sostanza, l'intero spettro delle relazioni transtestuali, tra interpretazione e consumo produttivo.

D'altra parte, il fruitore dei testi di culto intraprende la sua scorribanda interpretativa sulla base di caratteri pur sempre esibiti dal testo; e non sfugge ad Eco che, se i testi sgangherati lasciano l'iniziativa al lettore, altri testi faranno della sgangherabilità un principio generativo: «What Casablanca does unconsciously, other movies will do with an extreme intertextual awareness and with the expectation that the spectator be equally aware of their purposes. These are "postmodern" movies, where the quotations of the topos is recognized as the only way to cope with the burden of our encyclopedical filmic competence» (Eco 1985: 11). Gli esempi di Eco vanno dalla competenza da cinefili (la scalinata di Bananas/La corazzata Potëmkin), al dialogismo ironico (il duello in Raiders of the Lost Ark), alla competenza intermediale (l'incontro di personaggi disegnati da Rambaldi, uno proveniente da The Empire Strikes Back, durante il carnevale in E.T.). Sarebbe insomma lo stesso lettore modello di questi e molti altri testi a sollecitare una lettura scompaginata; si tratterebbe di una sorta di Lector excerpens che già preveda e guidi il riconoscimento, lo smontaggio, il godimento di singoli brani. Una catena di citazioni insistite

15 Isabella Pezzini invita poi a dedicare maggiore attenzione agli usi effettivi dei testi, per restare fedeli al mandato di Saussure: studiare i segni nella loro "vita sociale". L'esortazione è preziosa e andrebbe accolta e sviluppata. 


\section{Ocula ${ }^{22}$}

Vol 21, No 22 (April 2020) • DOI: 10.12977/ocula2020-5

Eduardo Grillo | Testi fatti a pezzi. Il culto mediale come ars excerpendi

consacrerebbe infine come "iconico" sia l'intero testo di partenza, sia ogni sua singola sezione.

Ma in definitiva, che siano "macchine consapevoli" o fortunati frutti del caso, le icone culturali sembrano guadagnarsi riconoscimento e venerazione non soltanto in virtù di condizioni storico-sociali, ma anche grazie a qualità testuali. Era questo il presupposto teorico delle riflessioni di Eco da cui si è partiti. I tratti del ri-uso degli archetipi intertestuali; il carattere pretestuale, ludico e immersivo del mondo rappresentato; una dialettica testo-lettore più libera e produttiva sono i caratteri testuali individuati in questo contributo che, condividendo quel presupposto, ha tentato di sviluppare quelle riflessioni.

\section{Bibliografia}

Altman, Rick

1999 Film/Genre, Bloomington, Indiana (In), Indiana University Press.

Brioschi, Franco; Di Girolamo, Costanzo

1984 Elementi di teoria letteraria, Milano, Principato.

Calabrese, Omar

1987 L'età neobarocca, Roma-Bari, Laterza; ora in Id., ll neobarocco. Forma e dinamiche della cultura contemporanea, Lucca, La Casa Usher, pp. 9-214.

Cavicchioli, Sandra

2002 "Spazio, descrizione, effetti di realtà", in Id., I sensi, lo spazio, gli umori e altri saggi, Milano, Bompiani, pp. 231-246.

Cevolini, Alberto

2006 De arte excerpendi. Imparare a dimenticare nella modernità, Firenze, Olschki Editore.

De Certeau, Michel

1980 L'Invention du quotidien, 1. : Arts de faire, Paris, Gallimard.

Eco, Umberto

1977 “Casablanca, o la rinascita degli dèi”, in Id., Dalla periferia dell'impero. Cronache da un nuovo medioevo, Milano, Bompiani, pp. 138-143 (già pubblicato come “Ore 9: Amleto all'assedio di Casablanca”, L'Espresso, 17 agosto 1975).

1979 Lector in fabula, Milano, Bompiani.

1985 “Casablanca: Cult Movies and Intertextual Collage”, SubStance, vol. 14, n. 2, issue 47, In Search of Eco's Roses, pp. 3-12 (tr. it. "Casablanca, cult movie e collage intertestuale”, in Guagnelini, Giovanni; Re, Valentina, 2007, pp. 172181).

1990 “Piccoli mondi”, in Id., I limiti dell’interpretazione, Milano, Bompiani, pp. 193-212.

1994 "Protocolli fittizi”, in Id., Sei passeggiate nei boschi narrativi, Milano, Bompiani, pp. 145-175.

1999 “Libri sgangherati”, in Id., La bustina di Minerva, Milano, Bompiani, pp. 239240. 


\section{Ocula}

Vol 21, No 22 (April 2020) • DOI: 10.12977/ocula2020-5

Eduardo Grillo | Testi fatti a pezzi. Il culto mediale come ars excerpendi

2007 “Dall'albero al labirinto", in Id., Dall'albero al labirinto. Studi storici sul segno e l'interpretazione, Milano, Bompiani.

Eibl, Karl

1998 "Textkörper und Textbedeutung. Über die Aggregatzustände von Literatur, mit einigen Beispielen aus der Geschichte des Faust-Stoffes", in Renate von Heydebrand (a cura di), Kanon Macht Kultur. Theoretische, historische und soziale Aspekte ästhetischer Kanonbildung, Stuttgart-Weimar, Metzler, pp. 60-77.

Fiorentino, Francesco

2009 "Icone culturali: per una definizione del concetto", in Id. (a cura di), Icone culturali d'Europa, Macerata, Quodlibet, pp. 9-24.

Frye, Northorp

1957 Anatomy of Criticism: Four Essays, Princeton (NJ), Princeton University Press (tr. it. Anatomia della critica. Quattro saggi, Torino, Einaudi, 2000).

Goodman, Nelson

1968 Languages of Art: An Approach to a Theory of Symbols, Indianapolis (IN), Hackett Publishing, 2a ed.

1978 Ways of worldmaking, Indianapolis (IN), Hackett Publishing.

Grillo, Eduardo

2012 "Culto e successo: il ruolo dell'intertestualità", in Bernardelli, Andrea (a cura di), La rete intertestuale. Percorsi tra testi, discorsi e immagini, Perugia, Morlacchi.

2016 "Note sulla serialità neobarocca, tra estetica e mitopoiesi”, Between. Rivista dell'Associazione di Teoria e Storia Comparata della Letteratura, Vol. VI, n. 11.

Guagnelini, Giovanni; Re, Valentina

2007 Visioni di altre visioni. Intertestualità e cinema, Bologna, ArchetipoLibri.

Jenkins, Henry

1992 Textual Poachers. Television Fans and Participatory Culture, London and New York (NY), Routledge.

Lausberg, Heinrich

1963 Elemente der literarischen Rhetorik. Eine Einführung für Studierende der klassischen, romanischen, englischen und deutschen Philologie, München, Max Hueber Verlag, $2^{\text {a }}$ ed (tr. it. Elementi di retorica, Bologna, il Mulino, 1969).

Maffesoli, Michel

2008 Iconologies: nos idol@tries postmodernes, Paris, Éditions Albin Michel (tr. it. Icone d'oggi: le nostre idol@trie postmoderne, Palermo, Sellerio, 2009).

Meletinskij, Eleazar M.

1994 O literaturnych archetipach, Moskva, RGGU (tr. it. Archetipi letterari, Macerata, eum, 2016, a cura di Massimo Bonafin).

Minzetanu, Andrei

2012 "La lecture citationnelle ou l'ars legendi comme ars excerpendi", Littérature, n. 168 , pp. 31-42. 


\section{Ocula ${ }^{22}$}

Vol 21, No 22 (April 2020) • DOI: 10.12977/ocula2020-5

Eduardo Grillo | Testi fatti a pezzi. Il culto mediale come ars excerpendi

Ortoleva, Peppino

2019 Miti a bassa intensità. Racconti, media, vita quotidiana, Torino, Einaudi.

Pezzini, Isabella; Sabucco, Veruska

2007 "Praticare il testo. Oltre l'interpretazione, gli usi", in Il testo galeotto, Roma, Meltemi, pp. 141-158.

Ryan, Marie-Laure

2015 Narrative as Virtual Reality 2. Revisiting Immersion and Interactivity in Literature and Electronic Media, Baltimore (MD), Johns Hopkins University Press.

Spaziante, Lucio

2006 "Conversazione a partire da Kurt Weill, su cultura popolare e mitopoiesi. Intervista a Umberto Eco”, $E / C$.

Truman, Emily

2017 "Rethinking the Cultural Icon: Its Use and Function in Popular Culture", Canadian Journal of Communication, Vol. 42, n. 5, pp. 829-845.

Eduardo Grillo è dottore di ricerca in Semiotica e comunicazione simbolica. Attualmente è docente di Semiotica dell'arte presso l'Accademia di Belle Arti di Napoli e presso L’Accademia di Belle Arti di Bologna; in precedenza ha insegnato Filosofia del linguaggio presso l'Università degli Studi di Perugia. Ha pubblicato, tra l'altro, Semiotica. Storia, teorie, metodi (con A. Bernardelli, Roma, Carocci, 2014) e Semiotica dell'investigazione (Roma, Carocci, 2014). Di recente ha avviato una linea di ricerca sulla tensione narrativa. 